\title{
Structural and magnetic anisotropy of directionally-crystallized ferromagnetic microwires
}

\author{
Svetlana Evstigneeva ${ }^{1}$, Alexander Morchenko ${ }^{1}$, Alexey Trukhanov ${ }^{1,2}$, Larissa Panina ${ }^{1,3}$, Vladimir Larin ${ }^{4}$, Natalia Volodina $^{1}$, \\ Nikolay Yudanov ${ }^{1}$, Makhsudsho Nematov ${ }^{1}$, Hisham Hashim ${ }^{1}$, and Haseeb Ahmad ${ }^{1}$ \\ ${ }^{1}$ NUST "MISIS", 119049 Moscow, Russia \\ ${ }^{2}$ Scientific Practical Materials Research Centre, NAS of Belarus, 220072 Minsk, Belarus \\ ${ }^{3}$ Institute for Design Problems in Microelectronics RAS, Moscow 124681, Russia \\ ${ }^{4}$ MFTI Ltd, MD2021 Kishinev, R. Moldova
}

\begin{abstract}
Amorphous ferromagnetic microwires have drawn attention primarily due to their excellent soft magnetic properties as the elements of sensors. In this work, semi-hard magnetic microwires of composition $\mathrm{Fe}_{4.3} \mathrm{Co}_{67.7} \mathrm{Si}_{11} \mathrm{~B}_{14} \mathrm{Cr}_{3}$ were obtained after a process of directional crystallization from amorphous state. The $\mathrm{XRD}$ analysis of crushed and whole wires identified the formation of the face-centered Co-modification as the main phase of directionally crystallized core alloy, which explains a large increase in anisotropy and coercivity. The application of a magnetic field during crystallization may orient the easy anisotropy axis of crystallites along the wire. This is confirmed by the investigation of crystallite orientation with respect to the wire axis and X-rays direction in diffractometer. The obtained results revealed that the formation of crystallites in amorphous Co-rich microwires occurs with the predominant orientation of the crystallographic direction [111] along the wire axis and the direction of a magnetic field during the directional crystallization process.
\end{abstract}

\section{Introduction}

Amorphous soft ferromagnetic wires exhibiting fast magnetization reversal and giant magnetoimpedance are promising materials for developing sensitive sensors of weak magnetic field, current, mechanical stress for various applications including structural health monitoring. In order to tailoring the operational magnetic parameters, the wires are subjected to different thermal treatments [1]: annealing in a magnetic field and/or under stress, current annealing, etc. After annealing treatment at temperatures close to the crystallization temperature, initially amorphous microwires change their structural and magnetic properties. Thus, the coercivity of Co-rich glass-coated microwires was significantly increased as a result of directional crystallization of the amorphous core [2-3].

The transformation from soft to hard magnetic properties was explained by the formation of crystallites of a hexagonal modification of cobalt [2] and their alignment along the wire during the crystallization process. A more detailed XRD analysis performed in [3] revealed the presence of face-centered modification of cobalt after the crystallization procedure, which could have cubic crystalline anisotropy. The coexistence of face-centered and hexagonal phases of nanocrystalline cobalt in $\mathrm{CoSiB}$ amorphous matrix was found in microwires, prepared by a special casting method [4].
In the present work, the mechanisms of coercivity and anisotropy increase in microwires after directional crystallization were investigated. A number of experiments with different orientation of the wire samples with respect to the measuring directions was carried out with the help of diffractometric and magnetometric methods.

\section{Experimental procedures}

Glass-coated amorphous microwires of the composition $\mathrm{Fe}_{4.3} \mathrm{Co}_{67.7} \mathrm{Si}_{11} \mathrm{~B}_{14} \mathrm{Cr}_{3}$ were used as initial materials. The metal core diameter and glass shell thickness were about 30 and $3.5 \mu \mathrm{m}$, respectively.

In order to form a nano- and/or microcrystalline structure, the wires were subjected to different annealing treatments as described in [2]. We consider that a directed crystallization may occur in the process of annealing at temperatures slightly lower than the primary crystallization temperature $T_{c r}$ when one end of microwires is overheated (by $\sim 60{ }^{\circ} \mathrm{C}$, named as "ignition") due to release of latent heat and propagation of the crystallization front. In accordance with the annealing procedure and resulting coercivity (estimated by the induction method), the wire samples were conditionally classified as explained in Table 1 .

Calorimeter DSC 204 F1 Netzsch was used to study the degree of crystallization of the obtained microwires. The sample heating was at the rate of $10 \mathrm{~K} / \mathrm{min}$ in an 
inert atmosphere. The values of $T_{c r}$ and the Curie temperature $T_{c}$ of the amorphous state estimated from DSC curves were $490{ }^{\circ} \mathrm{C}$ and $360{ }^{\circ} \mathrm{C}$, respectively. The value of $T_{c r}$ was used to set the annealing temperature $T_{\text {an }}$.

X-ray Diffractometer (DRON-3, Co K $\alpha$ - source) was used to obtain the diffraction patterns. The diffractogramms were detected with different orientation of the wire-samples relative to the X-ray direction. X-ray phase analysis was performed. In addition, Mössbauer spectra were used to study the magnetic texture in microwires.

Table 1. Annealing regimes and sample classification.

\begin{tabular}{|c|l|c|c|}
\hline Type & $\begin{array}{c}\text { The processing method and the } \\
\text { state of the wire after } \\
\text { processing }\end{array}$ & $\begin{array}{c}\text { Coercive } \\
\text { force } \\
\mathbf{H}_{\mathbf{c}}, \mathbf{O e} \\
\text { (induction } \\
\text { test) }\end{array}$ & $\begin{array}{c}\text { Coercive } \\
\text { force } \\
\mathbf{H}_{\mathbf{c}}, \mathbf{O e} \\
\text { (VSM } \\
\text { curves) }\end{array}$ \\
\hline 1 & Initial state (as-cast wires) & $0.2-0.6$ & $0.5-2.5$ \\
\hline 2 & $\begin{array}{l}\text { Crystallized by ordinary annealing } \\
\text { at } T_{a n}=560^{\circ} \mathrm{C}>T_{c r} \text { for } 10 \text { min }\end{array}$ & $\sim 12.5$ & $10-15$ \\
\hline 3 & $\begin{array}{l}\text { Not crystallized after ordinary } \\
\text { annealing at } T_{a n} \sim 485^{\circ} \mathrm{C}<T_{c r}\end{array}$ & $<12.5$ & $\sim 3$ \\
\hline 4 & $\begin{array}{l}\text { Not crystallized after annealing at } \\
T_{a n} \sim 485^{\circ} \mathrm{C}<T_{c r} \text { with ignition }\end{array}$ & $<12.5$ & $\sim 5$ \\
\hline 5 & $\begin{array}{l}\text { Directionally crystallized }\left(T_{a n}\right. \\
\sim 485^{\circ} \mathrm{C}<T_{c r} \text { with ignition) }\end{array}$ & $>>12.5$ & $20-90$ \\
\hline 6 & $\begin{array}{l}\text { Directionally crystallized }\left(T_{a n} \sim 485\right. \\
{ }^{\circ} \mathrm{C}<T_{c r} \text { with ignition in a magnetic } \\
\text { field of } 1 \mathrm{kOe})\end{array}$ & $>>12.5$ & $20-150$ \\
\hline 7 & $\begin{array}{l}\text { Partially crystallized }\left(T_{a n} \sim 4855^{\circ} \mathrm{C}\right. \\
<T_{c r} \text { with ignition in a magnetic } \\
\text { field of } 1 \mathrm{kOe})\end{array}$ & $<12.5$ & $10-20$ \\
\hline
\end{tabular}

The magnetization loops were measured with the help of vibrating sample magnetometer (modernized MV-07 and Liquid Helium "CryogenicLTD") for different wire orientation (along and perpendicular to the magnetizing field direction).

\section{Result and discussion}

Figure 1 presents the DSC curve for as-prepared microwires (type 1) in comparison with those for wires passed the directional crystallization (types 5 and 6) and partial directional crystallization (type 7). The DSC curve for as-prepared wire has two sharp peaks, indicating that the crystallization process from amorphous state proceeds in two stages. The wires after directional crystallization (type 5 and 6) are fully crystalline, because there are no sharp peaks in their DSC curves. The wires of type 7 passed only partial crystallization: there is only one peak in the DSC curve corresponding to the second crystallization stage. The DSC data for samples of types 2-4 are not presented as they are fully consistent with the crystalline state (flat curve) and quasi-amorphous state (broad peak).

Previosly $[2,3]$, the crystal structure of microwires was studied by X-ray diffraction methods. It was shown that cobalt is the main crystalline microphase in all samples. However, in contrast to [2], we found that this cobalt modification is the face-centered (fcc-Co) which was discussed in detail in [3].

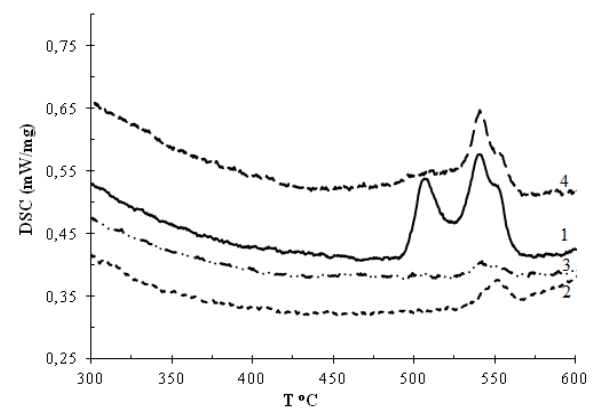

Fig. 1. DSC of glass coated microwires in various state: as-cast (1) and after directional crystallization: type 5 (2), type 6 (3), type 7 (4).

The XRD patterns for microwires after various annealing treatments are presented in Figs. 2-5. XRDspectra of as-prepared microwires are not shown as they display only the diffuse peak of the Co phase. The annealed microwires reveal the presence of $\mathrm{Co}, \mathrm{Fe}-\mathrm{Co}$, $\mathrm{CoSi}_{2}, \mathrm{Fe}-\mathrm{Si}$ and $\mathrm{Co}-\mathrm{B}$ nanosized phases. In Fig. 2, a sharp peak (associated with Co phase) is seen for microwires subjected to standard crystallization (type 2). In the case of wires of type 4 which failed the directional crystallization (Fig.3), the intensity of the peaks decreases and the estimated average size of Co-phase crystallites is in the range of 1.7-1.8 nm, which can be regarded as existence of a quasi-amorphous state. For the wires undergone directional crystallization (types 5), the average grain size of Co increases to 5.0-5.1 nm. In samples additionally subjected to a magnetic field during the directional crystallization, the crystallite size is slightly increased to $5.9-6.1 \mathrm{~nm}$.

The experiments with different arrangement of wirepieces in the X-ray apparatus relative to the axis of rotation (rocking) revealed the dependence of the XRD patterns on the wire position in the cell (Figs. 2-5).

It is seen that when scanning is performed with the location of the wire bundle along the beam of X-ray, additional peaks arise in the spectra of all the crystalline wires (for example, first reflex in Figs. 2-4 at the same angle $2 \theta$ position).

The spectra descend from top to bottom without deviations when angle is increasing, which indicates that the clusters are elongated rather than rounded, and there is a preferable orientation in the wire. The formation of crystallites of the dendrite type was also reported in [2]. The most obvious difference of the diffraction patterns obtained at various positions of the wires relative to the direction of the X-radiation is observed in samples of type 6 (Fig. 4) which were directionally crystallized in the presence of a large magnetic field, indicating the orientational influence of the field.

Additional weak peaks appearing at small reflection angles in the samples undergone directional crystallization (types 5-7), can presumably refer to $\mathrm{SiO}_{2}$ phases occurring in the glass shell and to the formation of superstructure with doubled lattice parameters (possibly, Fe-B, Co-B, Co-Si and Co-Si-B $[4,5]$ ). 


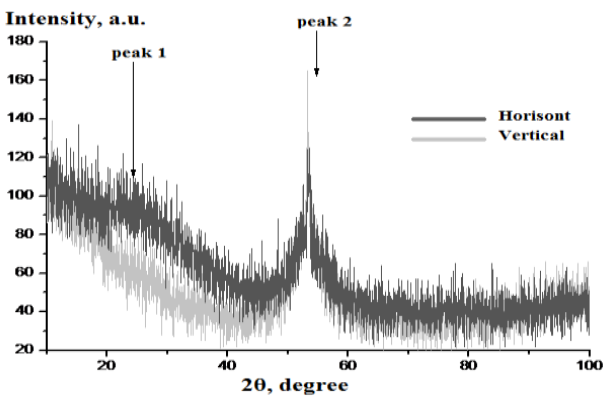

Fig. 2. XRD patterns for microwires of type 2 crystallised by standard annealing treatment $\left(T_{a n}=560^{\circ} \mathrm{C}>T_{c r}\right)$.

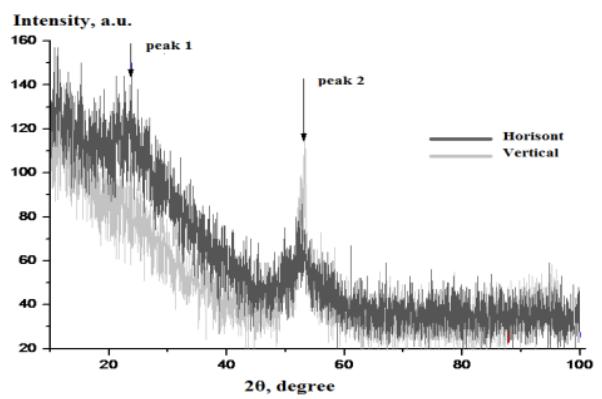

Fig. 3. XRD patterns for microwires of type 4 which failed the directional crystallization.

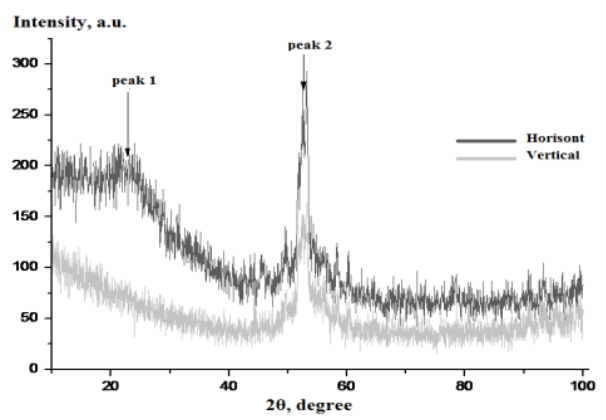

Fig. 4. XRD patterns for microwires after directional crystallization assisted by a dc magnetic field (type 6).

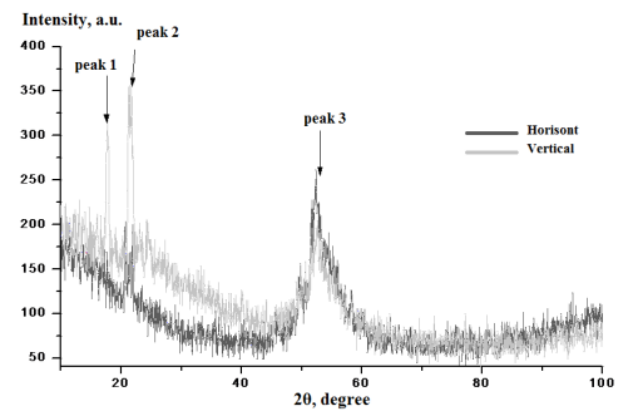

Fig. 5. XRD patterns for microwires after partial directional crystallization assisted by a dc magnetic field (type 7).

The factors that cause the preferential orientation of crystallites can be related to the magnetic field applied in the process of thermal treatment and the demagnetizing fields at temperatures lower than the Curie temperature $T_{c}$ (which considerably increases in the crystalline state). The other mechanism of oriented crystallization may be related with a change in elastic and magnetoelastic energies. In the process of directional crystallization, the clusters of the nascent crystalline Co phase under the action of a magnetic field are oriented along the wire axis. For these conditions, the total energy of the system will be lowered also by decreasing the magnetostatic energy. Since for cubic crystals with a negative constant of magnetocrystalline anisotropy $\left(K_{1}\right)$, the easy directions of magnetization are crystallographic axes of type $<111>$, the growing crystallites align their easy axes in the direction of the magnetic field vector, that is along the wire. Simultaneously, the other three axes of the same type will line up at the angle of $70.5^{\circ}$ to the wire axis (polar axis), and will be oriented with equal probability in different directions relative to the azimuth angle. Thus, a texture must be formed in the wire, which manifests itself in the appearance of additional complex anisotropy of a 'light cone' type. This assumption is consistent with the analysis of XRD patterns measured for different wire orientation relative to the X-rays direction.

The existence of magnetic texture is also supported by VSM measurements which were made for different wire positions with respect to the magnetizing field. Figure 6 presents the hysteresis loops of the as-prepared microwires for the two orientations. When the magnetizing field is parallel to the wire, the hysteresis loop is almost rectangular with the remanence close to saturation and very small coercivity of $0.5-2.5$ Oe. This confirms the existence of the axial anisotropy almost in the entire wire.

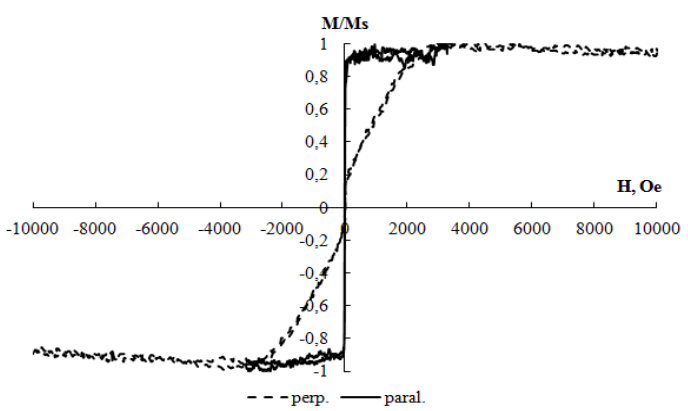

Fig. 6. Hysteresis loops of ferromagnetic microwire of type 1 with Rayleigh's correction.

In amorphous glass-coated microwires with positive magnetostriction, the axial magnetic anisotropy is formed due to a predominant axial tensile stress. In the surface layer, a radial closure domain structure may exist, but its effect on the total axial hysteresis is small. The remagnetization in the perpendicular field is described by an inclined curve with small hysteresis which could be due to transverse domain structure. Much higher saturation fields for perpendicular magnetization are caused by the demagnetization effect.

The effect of the perpendicular demagnetizing field on the magnetization can be eliminated applying the Rayleigh parabolic model of hysteresis [6]. The curve is shifted along the H-axis by the amount of the demagnetizing field. Then, the value of the average axial anisotropy constant $K_{\text {an }}$ can be estimated as the area between the hysteresis loop for positive values of the magnetizing field $\mathrm{H}$ and $\mathrm{y}$-axis [7]. The corrected 
magnetization curve for the wire sample of type 5 (directionally crystallized without a magnetic field) is given in Fig. 7. This curve is used to estimate the average axial anisotropy field which is found to be within 500-600 Oe. The wires directionally crystallized in the presence of the external field have larger anisotropy field in the range of $0.8-1 \mathrm{kOe}$ as found from corrected curve of Fig. 8. If the wires have only partial crystallization (type 7), the average anisotropy field drops below 100 Oe (see Fig. 9). The insert in Fig. 9 demonstrates the hysteresis shape for parallel magnetization which shows the presence of two magnetic phases with low $(<15 \mathrm{Oe})$ and high $(\sim 200 \mathrm{Oe})$ coercivities.

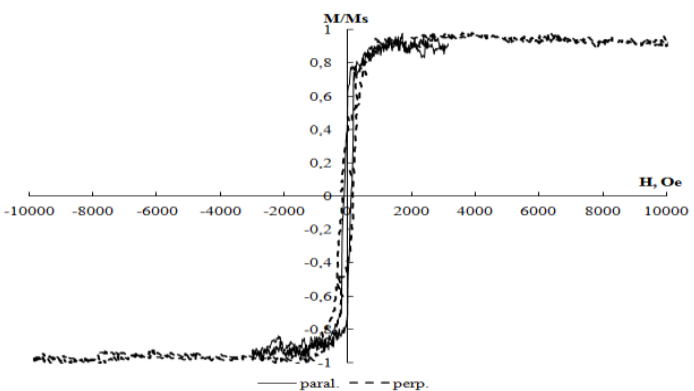

Fig. 7. Hysteresis loops of ferromagnetic microwire of type 5 with Rayleigh's correction.

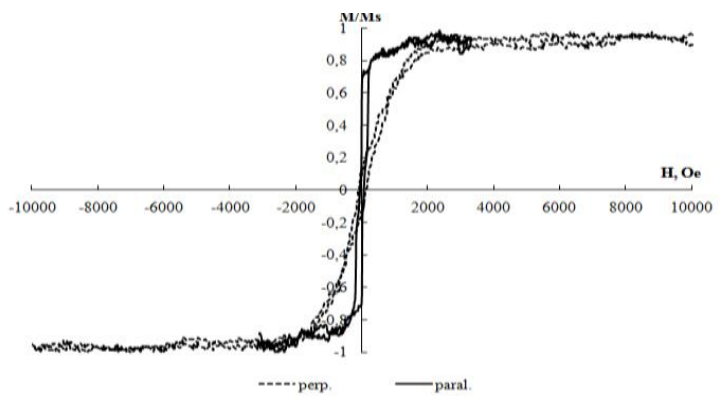

Fig. 8. Hysteresis loops of ferromagnetic microwire of type 6 with Rayleigh's correction.

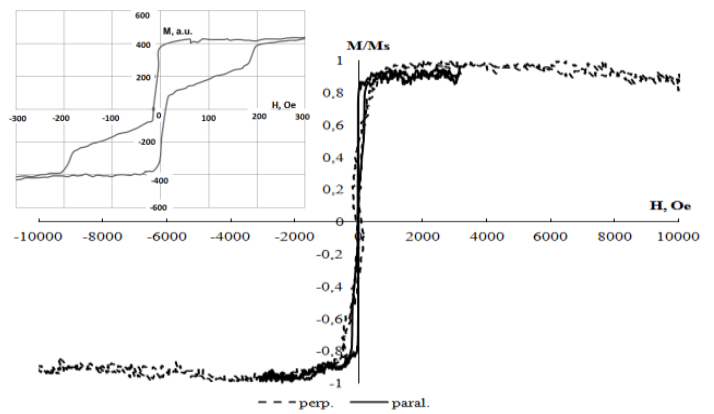

Fig. 9. Hysteresis loops of ferromagnetic microwire of type 7 with Rayleigh's correction. The inset shows an enlarged image of the hysteresis loop for parallel magnetization.

The estimated values of the anisotropy field $H_{K}$ can be compared with those due to the magnetocrystalline anisotropy of $<111>$ type (fcc-Co): $H_{K}=\left|4 K_{1}\right| /\left(3 M_{S}\right)$. Taking the magnetic parameters for crystalline Co: $K_{1}=$ $-700 \mathrm{kerg} / \mathrm{cm}^{3}$ and $M_{s}=1.5 \mathrm{kGs}[8]\left(T \ll T_{c}=\right.$ $1121^{\circ} \mathrm{C}$ ), the anisotropy field is about $620 \mathrm{Oe}$ at room temperature (and $\sim 430$ Oe at $T_{c r}$ ). This value is comparable with those estimated from Figs. 7, 8 and confirms the formation of predominately fcc-Co phase. A larger value of the anisotropy found for wires of type 6 may be due to additional contribution from induced strains.

Thus, we can estimate the average anisotropy field and the anisotropy constant of the crystallite matter. Comparing the obtained values with the literature data it is possible indirectly confirm the formation of a particular phase in the process of directional crystallization of ferromagnetic microwires.

\section{Conclusion}

It is established that the phase composition of initially amorphous ferromagnetic Co-enriched microwires changes as a result of their annealing treatment at the crystallization conditions. The main phase of the crystallized samples is the face-centered cobalt. Also it was found that the crystallites have an elongated shape and there exists a preferential orientation along the wire. This tendency is stronger in wires undergone a directional crystallization in the presence of a magnetic field.

The easy magnetization axes of crystallites correspond to the crystallographic directions of the $<111>$ type. Due to the effect of orientating action of the magnetic field, the crystallites gross direction coincides with one of the crystallographic direction [111], i.e. along the wire.

The magnetometry of the samples carried out for different orientations relative to the field direction of the electromagnet, made it possible to detect a noticeable anisotropy, which confirms the conclusion about the formation of a texture in the directionally crystallized wires and explains the appearance of hard magnetic state.

L. V. Panina acknowledges the support for this work under the Russian Federation State Contract for organising a scientific work (grant No 3.8022.2017).

\section{References}

1. Rodionova V., Chichay K., Zhukova V., et al., J. Supercond. Nov. Magn. 28, 977 (2015).

2. Larin V.S., Panina L.V., Patroi E. A., et al., Phys. Status Solidi (a) 213, 372 (2016).

3. Morchenko A. T., Panina L. V., Churyukanova M. N., et al., J. Alloys Compd. 698, 685 (2017).

4. Rodionova V., Baraban I., Chichay K., et al., Moscow International Symposium on Magnetism (MISM). Book of Abstracts, 466 (2017).

5. Životský O., Titov A., Jirásková Y., et al., J. Alloys Compd. 681, 402 (2016).

6. Bertotti G., Hysteresis in Magnetism (Academic Press, San Diego, 1998).

7. Herzer G. Acta Mater. 61, 718 (2013).

8. Takahashi M., Kim C.O., Koshimura M., Suzuki T., Jpn. J. Appl. Phys. 17, 1911 (1978). 